\title{
MDT - The Molecular Dynamics Test Set
}

\author{
Eric Barth \\ Department of Mathematics, Kalamazoo College, Kalamazoo MI,49006, USA, \\ barth@kzoo.edu
}

\begin{abstract}
Over the past two decades, computational scientists have turned increasing attention to the field of molecular modeling. Advances have been made in the design of efficient algorithms for problems such as fast summation methods for computing non-bonded atomic interactions, long-time numerical integration of equations of motion, non-Newtonian dynamical formulations for simulation in a variety of statistical mechanical ensembles, and optimization algorithms. These projects have often involved mathematicians and/or computer scientists who, though adept at algorithm and software development, may possess limited or no physical or chemical knowledge.

The molecular dynamics test set is a collection of model problems for aiding numerical analysts, code developers and others in the design of computational methods for molecular dynamics (MD) simulation. Common types of calculations and desirable features of algorithms have been considered, and these were used to guide selection of representative models. By including essential features of certain classes of molecular systems,

but otherwise limiting the physical and quantitative details, it is hoped that the test set will help to facilitate cross-disciplinary algorithm and code development efforts.
\end{abstract}

V.N. Alexandrov et al. (Eds.): ICCS 2001, LNCS 2074, p. 1065, 2001.

(c) Springer-Verlag Berlin Heidelberg 2001 